\title{
Influence of the Discretizing Method on the Identified Results in Boundary Value Inverse Analysis by the Boundary Element Method*
}

\author{
Shozo KAWAMURA**, Kennichi TAKAO**, \\ Hirofumi MINAMOTO** and Zahid HOSSAIN** \\ ${ }^{* *}$ Department of Mechanical Engineering, \\ Toyohashi University of Technology, \\ 1-1 Tempaku-cho, Toyohashi-shi, Aichi, 441-8580 Japan \\ E-mail: kawamura@mech.tut.ac.jp
}

\begin{abstract}
In this study, the influence of the discretizing method, such as a constant element or a linear element, on the accuracy of the identified results is investigated in the boundary value inverse analysis by the Boundary Element Method. For the regularization of the inverse analysis, the combination method is used; the one that the fundamental solution in B.E.M. is selected adequately and the one that the rank of the coefficient matrix is reduced. The optimum condition for solving the inverse problem is found by two performance indexes which are the condition number of the coefficient matrix and the residual norm caused by the rank reduction of the matrix. In a numerical example, the inverse problem governed by two-dimensional Laplace equation is treated. As a result, the identified result obtained using the linear element has almost the same accuracy as the one using the constant element while the accuracy using the constant element is often better, and the selection method of an adequate fundamental solution is very effective for the inverse analysis. Thus, the inverse analysis may be carried out using the constant element and the adequate fundamental solution selected.
\end{abstract}

Key words: Inverse Problem, Boundary Element Method, Optimum Design, Discretizing Method, Regularization, Fundamental Solution, Condition Number

\section{Introduction}

Recently, machine condition monitoring and diagnosis have become matters of great concern $^{(1)}$. In the case of diagnosis based on a mathematical model, the shape and property of the machine or structure, the governing equation, and the boundary and initial conditions have to be known. The identification of boundary conditions in the static problem is considered as a boundary value inverse problem. There have been many studies on this problems, and many kinds of subjects have been investigated ${ }^{(2)-(4)}$. When the boundary value inverse problem is solved using the Boundary Element Method (B.E.M.), the coefficient matrix derived by B.E.M. tends to be ill-posed. The noise included in the measured data has a great influence on the accuracy of the identified result. Many regularization methods have been proposed, such as rank reduction of the coefficient matrix based on the singular value decomposition, limitation of the solution space by applying constraints, Tikhonov regularization and a method using a Bayesian estimate and a Kalman

*Received 22 Nov., 2007 (No. T1-05-0245) Japanese Original : Trans. Jpn. Soc. Mech. Eng., Vol.72, No.713, A (2006), pp.24-31 (Received 9 Mar., 2005) [DOI: 10.1299/jcst.2.1] 
filter ${ }^{(5)}$. These regularization methods have been applied to the coefficient matrix of the constructed equation; however, it is possible to regularize the equation at the construction process. This idea has been proposed by one of the authors. Specifically, it is a method for selecting the satisfactory fundamental solution of B.E.M., and it has been applied to both the static problem ${ }^{(6)}$ and the dynamic one ${ }^{(7)}$. The basic idea is as follows. The shape of the boundary and the boundary condition for the fundamental solution are independent of the shape of the boundary and the boundary condition for the problem to be solved, so that various fundamental solutions can be adopted for the same governing equation. By changing the fundamental solution, the characteristics of the coefficient matrix can be changed. The possibility exists that for every problem to be solved, there is a satisfactory fundamental solution that can be adopted. However, in the case of 2-dimensional potential problem $^{(6)}$, the accuracy of the flux at salient points is not good because a constant element is used to discretize the potential and flux.

In this paper, the boundary value inverse problem for the analytical region with salient points is treated, and methods of selecting a satisfactory fundamental solution and reducing the rank of the coefficient matrix are used for regularization. The influence of the discretization method on the identified result is investigated. First, the boundary value inverse problem is formulated by B.E.M. Then for the 2-dimensional Laplace equation, a method is shown for changing the fundamental solution and the performance index, i.e., the condition number and the residual norm, to search for the optimum condition. As a numerical example, the identified results using the conventional fundamental solution and the proposed one are compared with respect to the discretization method.

\section{Formulation of boundary value problem by B.E.M.}

\subsection{Governing equation and boundary condition}

The governing equation without loading is given in the domain $\Omega$ using the linear differential operator $L$ as follows:

$$
L \cdot \phi=0 .
$$

The boundary conditions are of the Dirichlet type, $S(\phi)$, and Neumann type, $T(\phi)$.

\subsection{Boundary integral equation and the fundamental solution}

By using the usual B.E.M. procedure, the following equation can be obtained.

$$
\int_{\Omega}\left[\varphi \cdot L \phi-\phi \cdot L^{+} \varphi\right] d \Omega=\int_{\Gamma}\left[S^{+}(\varphi) T(\phi)-S(\phi) T^{+}(\varphi)\right] d \Gamma,
$$

where $\varphi$ is the trial function and $(\bullet)^{+}$is the adjoint operator.

For the function $\varphi$, the fundamental solution $\varphi^{*}$, which satisfies the equation

$$
L \varphi^{*}+\delta(\vec{x}-\vec{\xi})=0,
$$

is adopted, where $\vec{x}$ is the location of the observation point and $\vec{\xi}$ is the location of the source point.

Substituting Eqs.(1) and (3) into Eq.(2), the following equation can be obtained when $\vec{\xi}$ is in the domain $\Omega$.

$$
\phi(\vec{\xi})=\int_{\Gamma}\left[S^{+}\left(\varphi^{*}\right) T(\phi)-S(\phi) T^{+}\left(\varphi^{*}\right)\right] d \Gamma .
$$

The following equation can also be obtained when $\vec{\xi}$ is on the boundary $\Gamma$.

$$
c(\vec{\xi}) \phi(\vec{\xi})=\int_{\Gamma}\left[S^{+}\left(\varphi^{*}\right) T(\phi)-S(\phi) T^{+}\left(\varphi^{*}\right)\right] d \Gamma,
$$

where $c(\vec{\xi})$ is the coefficient determined from the shape of the boundary. 


\subsection{Discrete form of the integral equation}

In this study, the boundary value problem is investigated by considering Eq.(5). The boundary is divided into some elements, and the potential and flux are approximated by using a constant element, a linear element, and so on. In the previous study ${ }^{(6)}$, only a constant element was used, by which the potential and flux were both constant in an element so that no special treatment was needed even if the end of the element was a salient point. In the case of a direct problem, the linear element is often used to improve the accuracy of flux. Here, discretization by a linear element as used in the study is briefly explained. The discrete variables of potential and flux are set at both ends of an element. At a salient point, $S(\phi)$ is continuous but $T(\phi)$ is discontinuous, so two types of $T(\phi)$ are set there, i.e., the variable $T(\phi)$ at the right end of the left-side element of the salient point and that at the left end of the right-side element of the salient point. Therefore, when there are four salient points in the analytical region, for example, the number of variable $T(\phi)$ is four greater than that of variable $S(\phi)$.

Using a constant element or a linear one, the discrete form of Eq.(5) is as follows:

$$
[H]\{S(\phi)\}-[G]\{T(\phi)\}=\{0\} .
$$

The equation is reconstructed to form a simultaneous algebraic equation with respect to the unknown variables as follows:

$$
[D]\{p\}=\{g\},
$$

where $[D]$ is the coefficient matrix, $\{p\}$ is a vector composed of the unknown boundary values and $\{g\}$ is the vector calculated from the given boundary values.

The boundary value inverse problem is the case when both $S(\phi)$ and $T(\phi)$ are unknown on some boundaries. Then the coefficient matrix $[D]$ becomes ill-posed so that a regularization procedure is needed. In this study, methods of selecting a satisfactory fundamental solution and reducing the rank of the coefficient matrix are used for regularization.

\section{Fundamental solution with various parameters}

The fundamental solution $\varphi^{*}$ which satisfies Eq.(3) is the value of $\varphi$ when the unit potential is applied in the domain $\Omega$ governed by the linear differential operator $L$. In this case, the shape of the boundary and the boundary condition for the fundamental solution can be determined so as to be independent of the shape of the boundary and the boundary condition for the problem to be solved. Thus, various fundamental solutions can be adopted for the same governing equation ${ }^{(8)}$.

In this study, the 2-dimensional Laplace equation is used. The fundamental solution is shown as follows.

The normalized governing equation is expressed as follows:

$$
\nabla^{2} u=0 \text {. }
$$

The fundamental solution, which is generally used, is expressed as follows:

$$
u^{*}(r)=\frac{1}{2 \pi} \ln \left(\frac{1}{r}\right),
$$

where $r$ is the distance between the observation point and the source point.

Here the 2-dimensional Laplace equation is considered as the equation which governs the deformation of the membrane. So, as the fundamental solution, the deformation function when the unit load is applied at the center of the membrane of the radius $R$, i.e.,

$$
u^{*}(r)=\frac{1}{2 \pi} \ln \left(\frac{R}{r}\right)
$$


can be adopted.

At this time, the radius $R$ is independent of the problem to be solved so that various fundamental solutions can be considered for various values of $R$.

\section{Evaluation of optimum condition for inverse analysis}

In this study, the coefficient matrix $[D]$ in Eq.(7) is constructed using the fundamental solution with parameter $R$ in Eq.(10). The rank of $[D]$ is reduced to $k$ by neglecting the small singular values resulting in a new matrix $\left[D_{k}(R)\right]$. The characteristics of $\left[D_{k}(R)\right]$ are evaluated by two indices as follows.

First is the condition number, $\operatorname{Cond}_{k}(R)$, defined as follows :

$$
\operatorname{Cond}_{k}(R)=\lambda_{\max } / \lambda_{\min },
$$

where $\lambda_{\max }, \lambda_{\min }$ are the maximum and minimum singular values of $\left[D_{k}(R)\right]$. The condition number means the maximum expansion ratio of the relative error including in $\{g\}$.

Second is the error norm, $E_{k}(R)$, defined as follows :

$$
E_{k}(R) \equiv\left\|[D]\left\{p_{k}(R)\right\}-\{g\}\right\|,
$$

where $\left\{p_{k}(R)\right\}$ is the identified boundary value vector using the coefficient matrix $\left[D_{k}(R)\right]$. This index evaluates the worse of identification accuracy by reducing the rank of the coefficient matrix.

The optimum condition for inverse analysis is determined synthetically using these two indices.

\section{Check the influence of the discretization method by numerical example}

\subsection{Analytical model and boundary conditions}

The rectangular domain of size $2.0 \times 1.0$ shown in Fig. 1 is considered. Each side is divided uniformly into 10 elements. Two types of boundary conditions are set as shown in Table 1. For $\Gamma_{3}$, both the potential $u$ and flux $q(=\partial u / \partial n)$ are unknown.

\subsection{Results in boundary condition of 'case 1'}

First, the optimum inverse analysis condition is evaluated in the case of $R=1.0$, which is the conventional fundamental solution. Using a constant element or a linear element, the changes of performance indices are shown in Fig.2 for rank $k$.

There have been several studies determining the optimum inverse analysis condition, especially, the optimum rank $k$ in this numerical example. Because the exact values are actually unknown, the optimum condition has to be determined by experience or trial simulation. Based on another group's study ${ }^{(4)}$ and our own ${ }^{(6),(7)}$, the optimum condition of rank $k$ is about 30 because both $u$ and $q$ are unknown at 10 nodes. In this study, the optimum condition may be determined from the condition number; that is, the condition

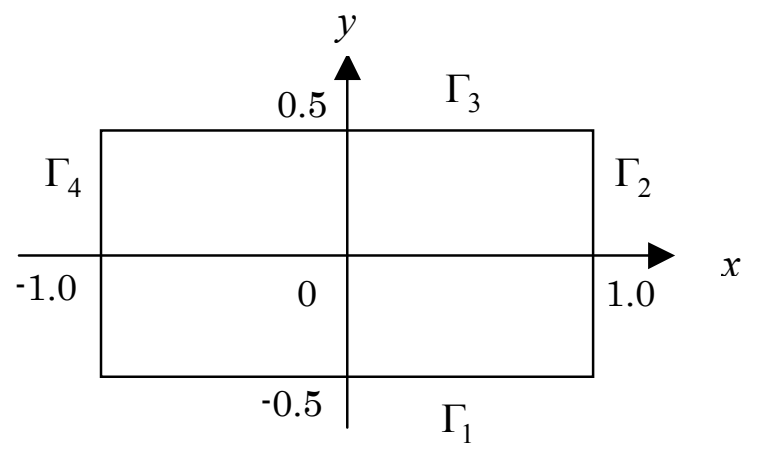

Fig.1 Model for analysis 
(a) case 1

\begin{tabular}{|c|c|c|}
\hline & $u$ & $q$ \\
\hline$\Gamma_{1}$ & 1.0 & -1.0 \\
\hline$\Gamma_{2}$ & unknown & 0 \\
\hline$\Gamma_{3}$ & unknown & unknown \\
\hline$\Gamma_{4}$ & unknown & 0 \\
\hline
\end{tabular}

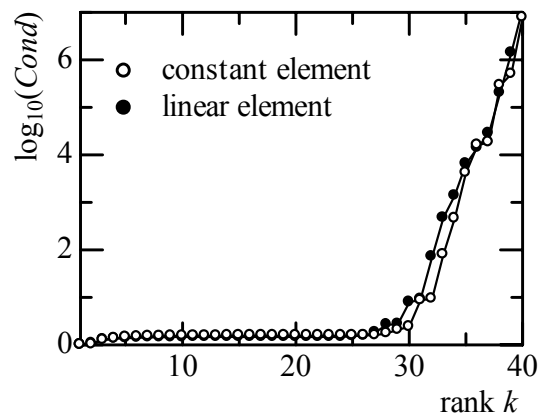

(a) Condition number

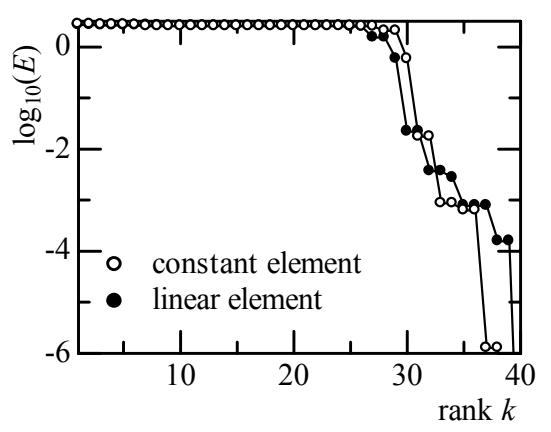

(b) Residual norm

Fig.2 Performance index for case 1 and $R=1.0$ (b) case 2

\begin{tabular}{|c|c|c|}
\hline & $u$ & $q$ \\
\hline$\Gamma_{1}$ & 1.0 & -1.0 \\
\hline$\Gamma_{2}$ & $\begin{array}{c}\text { linearly change } \\
1.0-2.0\end{array}$ & unknown \\
\hline$\Gamma_{3}$ & unknown & unknown \\
\hline$\Gamma_{4}$ & $\begin{array}{c}\text { linearly change } \\
2.0-1.0\end{array}$ & unknown \\
\hline
\end{tabular}

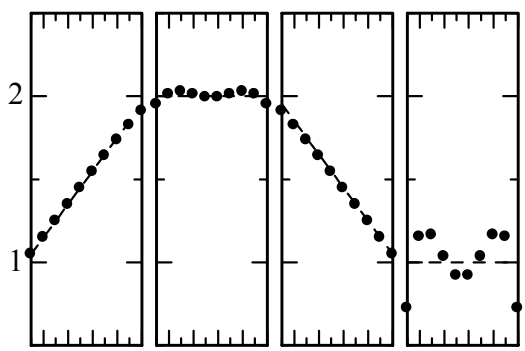

(A)

(B)

(C)

(D)

(a) the case using the constant element

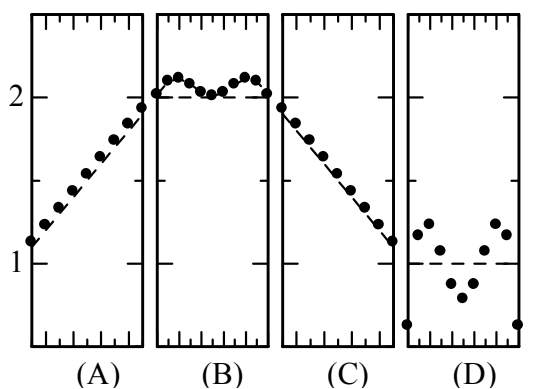

(b) the case using the linear element

Fig.3 Identified result at optimum condition for case 1 and $R=1.0$ (A) $u$ on $\Gamma_{2}$, (B) $u$ on $\Gamma_{3}$, (C) $u$ on $\Gamma_{4}$, (D) $q$ on $\Gamma_{3}$

number is larger in the range under 2.0 in the log scale at the optimum rank.

Using this design criteria, the optimum inverse analysis condition is $k=33$ in the case of a constant element as well as $k=32$ in the case of a linear element. From Fig.2(a) depicting the condition number, the indices are almost the same, such as 1.90 in the log scale in the case of a constant element and 1.85 in the case of a linear element. But from Fig 2(b) depicting the residual norm, the index is -3.06 and -2.44 , respectively, so that the performance in the case of a constant element is better than that in the case of a linear element.

The identified result at the optimum condition is shown in Fig.3. Figure 3(A), (B) and (C) show the results of $u$ on $\Gamma_{2}, \Gamma_{3}$ and $\Gamma_{4}$, and Fig.3(D) shows that of $q$ on $\Gamma_{3}$. To estimate the results quantitatively, an error index is introduced as follows:

$$
\sqrt{\sum(\text { identified results/exact results }-1)^{2}} \text {, }
$$

and the result is shown in Table 2. This method also shows that the performance in the case of a constant element is better than that in the case of a linear element. It is noted that using 
the linear element leads to good accuracy in direct analysis but not always in inverse analysis.

From here, the selecting method of a satisfactory fundamental solution is applied as Table 2 Error of identified results

\begin{tabular}{|c|c|c|c|c|}
\hline & $(\mathrm{A})$ & $(\mathrm{B})$ & $(\mathrm{C})$ & $(\mathrm{D})$ \\
\hline $\begin{array}{c}\text { Constant element } \\
R=1.0, \quad k=33\end{array}$ & $2.7 \%$ & $4.1 \%$ & $2.7 \%$ & $51.8 \%$ \\
\hline $\begin{array}{c}\text { Linear element } \\
R=1.0, \quad k=32\end{array}$ & $7.0 \%$ & $12.1 \%$ & $7.0 \%$ & $72.9 \%$ \\
\hline $\begin{array}{c}\text { Constant element } \\
R=0.8, \quad k=33\end{array}$ & $1.3 \%$ & $2.0 \%$ & $1.3 \%$ & $19.3 \%$ \\
\hline $\begin{array}{c}\text { Linear element } \\
R=2.0, k=32\end{array}$ & $1.8 \%$ & $7.6 \%$ & $1.8 \%$ & $98.6 \%$ \\
\hline
\end{tabular}

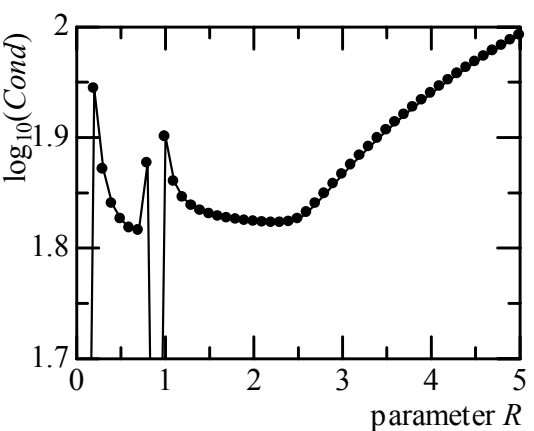

(a) Condition number

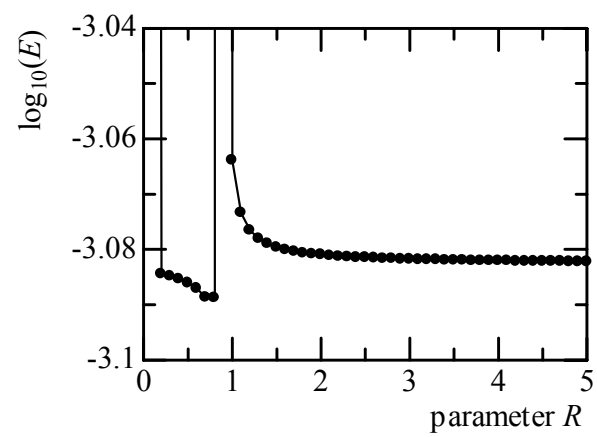

(b) Residual norm

Fig.4 Performance index at optimum condition for case 1 and every parameter $R$ using the constant element

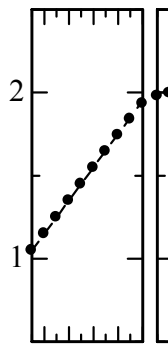

(A)

Fig.5 Identified result at optimum condition for case 1 using the constant element

(A) $u$ on $\Gamma_{2}$, (B) $u$ on $\Gamma_{3}$,

(C) $u$ on $\Gamma_{4}$, (D) $q$ on $\Gamma_{3}$

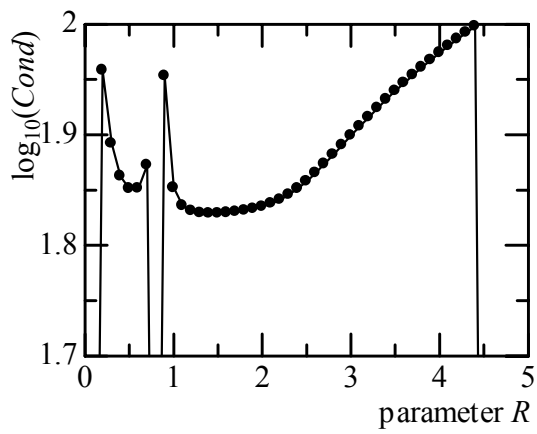

(a) Condition number

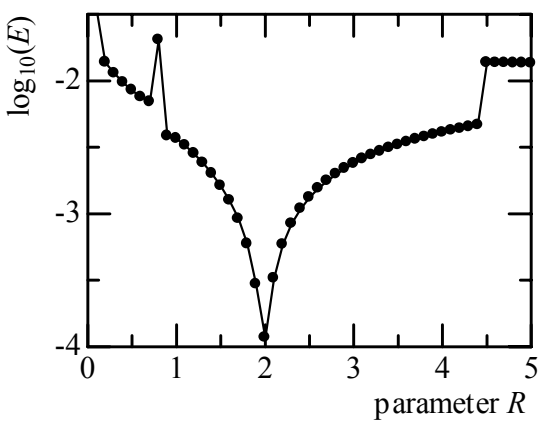

(b) Residual norm

Fig.6 Performance index at optimum condition for case 1 and every parameter $R$ using the linear element

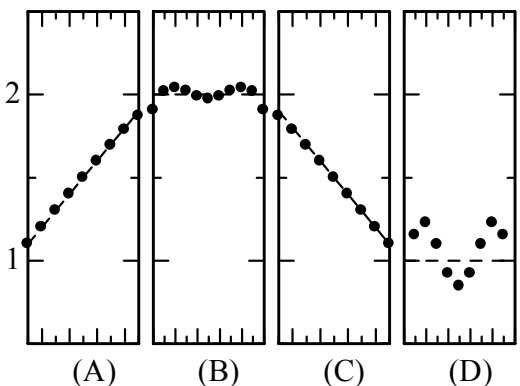

Fig.7 Identified result at optimum condition for case 1 using the linear element (A) $u$ on $\Gamma_{2}$, (B) $u$ on $\Gamma_{3}$, (C) $u$ on $\Gamma_{4}$, (D) $q$ on $\Gamma_{3}$ 
follows.

(1) Parameter $R$ is changed from 0.1 to 5.0 .

(2) After parameter $R$ is set as a certain value with interval 0.1, the condition number and residual norm are calculated when the rank $k$ of the coefficient matrix is changed.

(3) The optimum rank $k$ for the parameter $R$ is determined such that the condition number is larger in the range under 2.0 in the log scale.

(4) The optimum rank is determined for every parameter $R$, and the condition number and residual norm are obtained for every parameter $R$ and the optimum rank $k$.

(5) The optimum inverse analysis condition is determined where the residual norm is the smallest.

The condition number and residual norm are shown in Fig.4 in the case of a constant element. The residual norm is very large at $R=0.9$, because the optimum rank is small within the constraint condition, and the identified result differs from the exact result. A similar phenomenon can be seen in other results.

From Fig.4(b), the optimum condition is $R=0.8$ because the residual norm is a minimum. At this time, the rank $k=33$, so the optimum condition is $R=0.8$ and $k=33$. The identified result is shown in Fig.5 and the error index is shown in Table 2. The improvement of the identified result can be seen by comparing it with Fig.3(a).

The results shown in Fig. 6 represent the case of a linear element. The optimum condition is $R=2.0$ and $k=32$. The identified result is shown in Fig.7 and the error index is shown in Table 2. The potential $u$ is improved in (A), (B) and (C) but the flux $q$ is not improved in comparison with Fig.3(b).

It is very effective that the optimum condition can be predicted from the coefficient matrix, but it is difficult now and it may be the topic of a future work.

\subsection{Results in boundary condition of 'case 2'}

As in Sec 5.2, the optimum inverse analysis condition is first evaluated in the case of $R=1.0$. Using a constant element or a linear element, the changes of performance indices for rank $k$ are shown in Fig.8. There are some elements where the potential $u$ is known so that some flux $q$ at salient points can be calculated. For example, the potential $u$ on $\Gamma_{2}$ is known so that the flux $q$ at the salient point on $\Gamma_{3}$ can be calculated as (-1.0). Therefore the number of unknown variables is 38 and the full rank of the coefficient matrix in the case of a linear element is 38 in Fig.8. The optimum rank is determined from the condition number; that is, the index approaches 2.0 but remains less than 2.0.

Using this design criteria, the optimum inverse analysis condition is $k=32$ in the case of a constant element as well as $k=31$ in the case of a linear element. From Fig.8(a) depicting the condition number, the indices are almost same, such as 1.80 in the case of a constant element and 1.75 in the case of a linear element. But from Fig 8(b) depicting the residual norm, the index is -2.8 and -1.7 , respectively, so that the performance in the case of a constant element is better than that in the case of a linear element.

\begin{tabular}{|c|c|c|c|c|}
\hline & Table 3 Error of identified results \\
\hline $\begin{array}{c}\text { Constant element } \\
R=1.0, \quad k=32\end{array}$ & $7.4 \%$ & 0.55 & $74.0 \%$ & 0.55 \\
\hline $\begin{array}{c}\text { Linear element } \\
R=1.0, \quad k=31\end{array}$ & $18.4 \%$ & 0.36 & $117 \%$ & 0.36 \\
\hline $\begin{array}{c}\text { Constant element } \\
R=3.4, \quad k=31\end{array}$ & $7.7 \%$ & 0.59 & $78.2 \%$ & 0.59 \\
\hline $\begin{array}{c}\text { Linear element } \\
R=2.1, k=31\end{array}$ & $9.5 \%$ & 0.47 & $89.6 \%$ & 0.47 \\
\hline
\end{tabular}


The identified results at the optimum condition are shown in Fig.9 and the error index is shown in Table 3. Here in (B) and (C), the exact results are zero so that the error index is defined as

$$
\sqrt{\sum(\text { identified results })^{2}}
$$

From Fig.9 and Table 3, the potential $u$ in (A) and flux $q$ in (C) can be identified accurately in the case of a constant element, while in the case of a linear element, the flux $q$ in (B) and (D) is accurate. Checking the accuracy at a salient point between (B) and (C), the identified result agrees well in (B) in the case of a constant element, while in the case of a linear element, it agrees well in (C). It is also noted in 'case 2' that using the linear element leads to good accuracy in the direct analysis but not always in the inverse analysis.

Next, the selection method of a satisfactory fundamental solution is applied, as in the previous section.

The condition number and residual norm are shown in Fig.10 in the case of a constant element. From Fig.10(b), the optimum condition is $R=1.0$, so good identification accuracy can be obtained using the conventional fundamental solution. In Fig.10(a), it is observed that the condition number is a minimum at $R=3.4(k=31)$ and the residual norm is almost the same as that of $R=1.0$. The identified result at this condition is shown in Fig.11, and the error index is also shown in Table 3. The result is almost the same as that of Fig.9(a), and the expansion ratio of the relative error is small because the condition number is small.

The results in the case of a linear element are shown in Fig.12. The optimum condition is $R=2.1$ and $k=31$. The identified result is shown in Fig. 13 and the error index is shown in Table 3. The potential $u$ in (A) and flux $q$ in (C) shows improvement compared with the result in Fig.9(b).

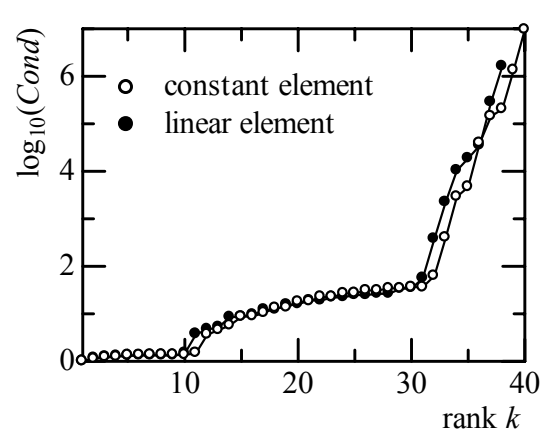

(a) Condition number

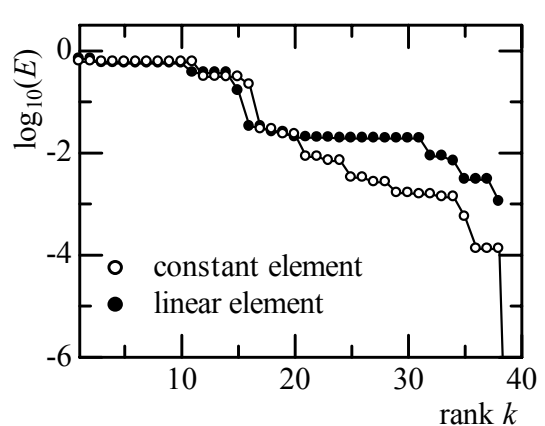

(b) Residual norm

Fig.8 Performance index for case 2 and $R=1.0$

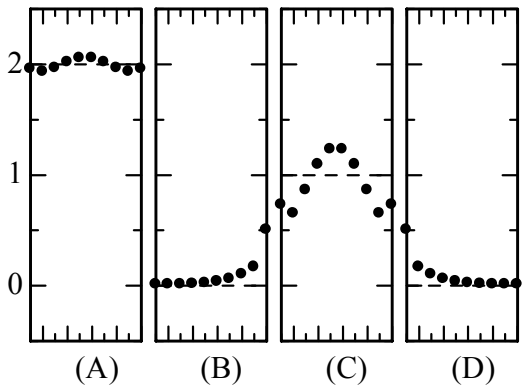

(a) the case using the constant element

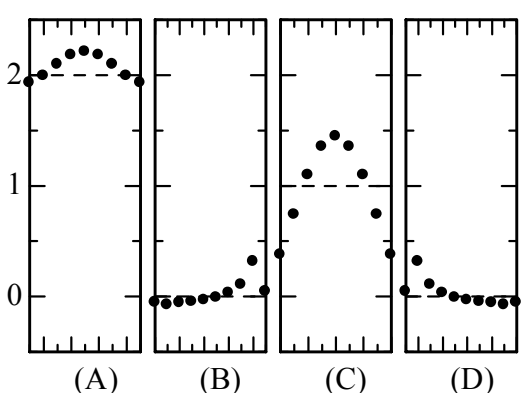

(b) the case using the linear element

Fig.9 Identified result at optimum condition for case 2 and $R=1.0$

(A) $u$ on $\Gamma_{3}$, (B) $q$ on $\Gamma_{2}$,

(C) $q$ on $\Gamma_{3}$, (D) $q$ on $\Gamma_{4}$ 


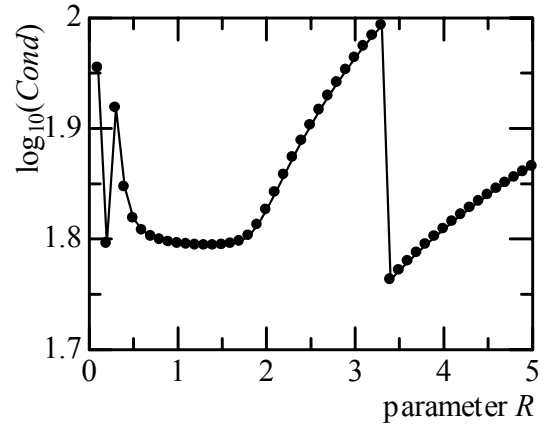

(a) Condition number

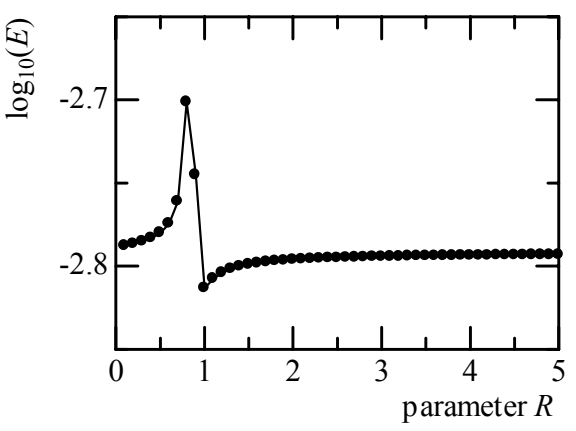

(b) Residual norm

Fig.10 Performance index at optimum condition for case 2 and every parameter $R$ using the constant element

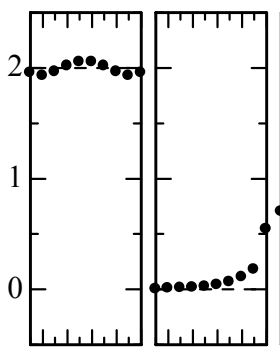

(A)

(B)

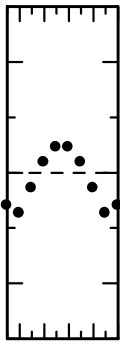

(C)

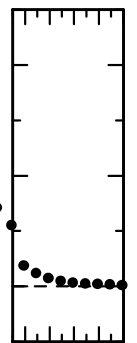

(D)
Fig.11 Identified result at another optimum condition for case 2 using the constant element

(A) $u$ on $\Gamma_{3}$, (B) $q$ on $\Gamma_{2}$,

(C) $q$ on $\Gamma_{3}$, (D) $q$ on $\Gamma_{4}$

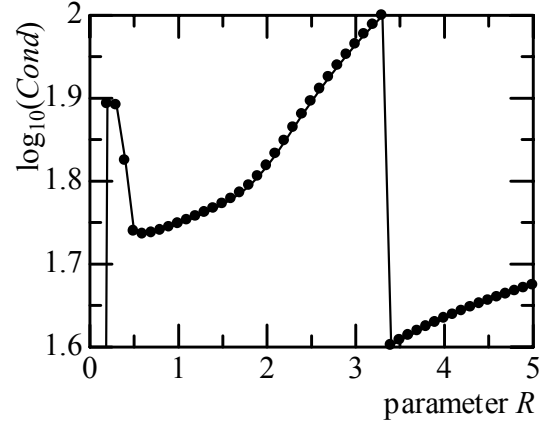

(a) Condition number

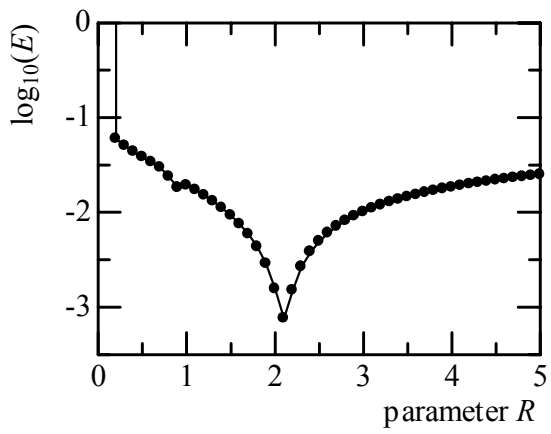

(b) Residual norm

Fig.12 Performance index at optimum condition for case 2 and every parameter $R$ using the linear element

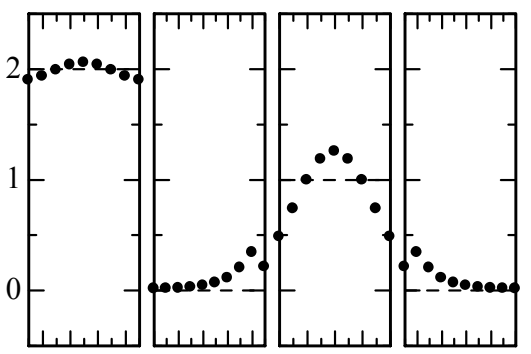

(A)

(B)

(C)

(D)

Fig.13 Identified result at optimum condition for case 2 using the linear element (A) $u$ on $\Gamma_{3}$, (B) $q$ on $\Gamma_{2}$,

(C) $q$ on $\Gamma_{3}$, (D) $q$ on $\Gamma_{4}$

\subsection{Discussions}

In the direct analysis of an analytical region with salient points, the accuracy can be generally improved using the linear element to discretize the potential and flux. In this study, the effect of the linear element in the inverse analysis is checked in terms of whether the accuracy by the linear element is the same as or worse than that by the constant element. This shows that some adjustments to improve the accuracy in direct analysis do not work well for inverse analysis. It is well known that good accuracy can be obtained by using more elements of the boundary in direct analysis, but the accuracy will worsen in the inverse analysis because the condition number will become large. Similarly, a linear element doesn't lead to high accuracy in the inverse analysis. Also, the combined methods of selecting a satisfactory fundamental solution and reducing the rank of the coefficient matrix are very effective in the inverse analysis compared with the identified results using 
the conventional fundamental solution.

\section{Conclusions}

In this paper, the boundary value inverse problem for an analytical region with salient points is discussed, and methods of selecting a satisfactory fundamental solution and reducing the rank of the coefficient matrix are used for regularization. The influence of the discretization method on the identified results is investigated.

As a result, the accuracy by the linear element is the same as or worse than that by the constant element. This shows that some adjustments to improve the accuracy in direct analysis do not work well for inverse analysis. Also, the combined methods of selecting a satisfactory fundamental solution and reducing the rank of the coefficient matrix are very effective in inverse analysis compared with the identified results using the conventional fundamental solution.

\section{References}

(1) Raj Rao(Eds.), Proc. 16th International Congress and Exhibition on Condition Monitoring and Diagnostic Engineering Management, 2003.

(2) Japan Society of Mechanical Engineers, Computer Analysis of Inverse Problems (in Japanese), 1991, Corona Publishing Co., Ltd.

(3) S.Kubo, Inverse Problem (in Japanese), 1992, Baifukan Co., Ltd.

(4) S.Kubo, S.Kuwayama and K.Ohji, Mathematical Structure of Numerical Analysis and Regularization of an Inverse Boundary Value Problem in Laplace Field (in Japanses), Transactions of the JSME, Vol.61, No.581, A, 1995, pp.169-176.

(5) A. Murakami, N. Tosaka, M. Hori and M. Suzuki, Inverse Analysis with FEM/BEM, Application of the Kalman Filter and the Equivalent Inclusion Method (in Japanese), 2002, Corona Publishing Co., Ltd.

(6) S. Kawamura, T. Ibuki and T. Iwatsubo, Selection of Satisfactory Fundamental Solution in Boundary Value Inverse Analysis by B.E.M., JSME International Journal, Vol.42, No.3, A, 1999, pp.342-347.

(7) S. Kawamura, M. Unigame and T. Iwatsubo, Estimation of Machine Vibration from Acoustic Measurements (Proposition of Regularization Method in Inverse Analysis of Acoustic-Vibratory Systems) (in Japanses), Transactions of the JSME, Vol.66, No.652, C, 2000, pp.3911-3918.

(8) Japan Society for Computational Methods in Engineering, Theory and Applications in Boundary Element Method (in Japanese), 1986, p.72, Corona Publishing Co., Ltd. 